\title{
Détection d'antigènes circulants au cours d'une infection expérimentale à Trypanosoma brucei brucei chez des bovins Borgou, Lagunaire et zébus Bororo blancs
}

\author{
A. Doko ${ }^{1,2}$ A. Verhulst 1 V.S. Pandey ${ }^{1}$ * \\ Ph. Büscher ${ }^{1}$ V. Lejon ${ }^{1}$
}

Mots-clés

Bovin Borgou - Bovin des Lagunes Zébu White Fulani - Trypanosoma brucei brucei - Infection expérimentale - Test ELISA - Antigène Bénin.

\begin{abstract}
Résumé
Des bovins Borgou $(n=10)$, Lagunaire $(n=10)$ et zébus Bororo blancs $(n=10)$ du Bénin ont été infectés expérimentalement avec un clone de Trypanosoma brucei brucei en vue d'évaluer le test ELISA de détection d'antigènes circulants. Aucun des trois témoins non inoculés de chacune des races n'a développé d'infection. Les bovins Lagunaire et Borgou ont développé une maladie bénigne suivie de guérison spontanée, les zébus Bororo blancs une maladie typique à allure chronique et fatale après plusieurs mois. Pour tous les animaux confondus, la sensibilité du test de détection des antigènes circulants de $T$. brucei a été de 20,46 p. 100, nettement inférieure à celle de la détection des parasites par la méthode du buffy coat $(40,24$ p. 100$)$. La sensibilité du test varie fortement en fonction du type d'animaux infectés et de l'allure de l'infection ; elle est la plus élevée chez les bovins Borgou aux parasitémies élevées mais de durée plus courte $(44,44$ p. 100$)$ et la plus faible chez les zébus Bororo blancs aux parasitémies faibles et intermittentes $(4,09$ p. 100). Augmenter la sensibilité du test de détection des antigènes circulants en abaissant la densité optique de 0,050 (seuil de positivité) à 0,025 paraît impossible sans altérer sa spécificité. Comparés au buffy coat, les faux négatifs du début d'infection et les faux positifs d'après la guérison spontanée compromettent également la valeur du test pour le diagnostic des infections actives à T. brucei.
\end{abstract}

\section{INTRODUCTION}

Décrite pour les trypanosomoses animales par Murray et coll. (7), la technique du buffy coat est souvent utilisée comme référence pour évaluer la sensibilité d'autres techniques de diagnostic des infections actives. Mais certains animaux présentent des parasitémies faibles ou fluctuantes difficiles à mettre en évidence par les tests parasitologiques classiques. Devant ces contraintes, des tests visant à détecter les anticorps trypanosomiens ont été développés, tels l'immunofluorescence indirecte (IFI) et l'enzyme linked immunosorbent assay (ELISA). Ces tests ont également leur limite :

\footnotetext{
1. Institut de Médecine Tropicale Prince Léopold, Nationalestraat 155 , 2000 Antwerp, Belgique

2. Faculté des Sciences Agronomiques, Université Nationale du Bénin BP 526, Cotonou, Bénin

* Pour la correspondance : fax : 32321614 31, E-mail : animprod@vet.itg.be
}

ils permettent de détecter un contact antérieur avec le trypanosome mais ne permettent pas de distinguer une infection active d'une infection guérie. Les animaux porteurs d'anticorps trypanosomiens doivent être considérés comme des réservoirs potentiels de la maladie (4). Le test de détection des antigènes circulants de trypanosomes (10) pourrait offrir une alternative pour la détection des infections actives. Ce test a déjà été évalué en Afrique de l'Ouest lors d'infections naturelles et expérimentales à Trypanosoma congolense et T. vivax (6). Sa sensibilité était de 63 p. 100 pour $T$. congolense, mais seulement de 10 p. 100 pour T. vivax. Toutefois, les infections à $T$. brucei peuvent également avoir un impact économique non négligeable chez les bovins en Afrique de l'Ouest (4). Le diagnostic parasitaire est difficile dans les affections chroniques, d'autant plus que les parasites peuvent être peu nombreux dans le sang périphérique en raison de leur prédilection tissulaire. Le but du présent travail est d'apprécier la valeur diagnostique de ce test au cours d'une infection expérimentale à $T$. brucei brucei chez des bovins de races Borgou, Lagunaire et zébu Bororo blanc au Bénin. 


\section{MATERIEL ET METHODES}

\section{Animaux}

L'expérience a porté sur 39 bovins mâles et femelles adultes, appartenant aux races Borgou ( $\mathrm{n}=13$, dont 10 infectés et 3 témoins non infectés), Lagunaire ( $\mathrm{n}=13$, dont 10 infectés et 3 témoins non infectés) et zébu Bororo blanc ( $\mathrm{n}=13$, dont 10 infectés et 3 témoins non infectés). Ces animaux, originaires de régions à trypanosomose endémique, avaient été transférés à la ferme de l'université à Cotonou où le risque trypanosomien était quasi nul au moment de l'expérimentation. Un mois avant l'expérience, tous les animaux avaient été vermifugés au thiabendazole et traités à 똗 l'acéturate de diminazène (Berenil, Hoechst). Au moment de l'infection expérimentale, tous les bovins étaient négatifs à la technique du buffy coat, au test de détection des antigènes circulants de T. brucei et au test de lyse spécifique du variant AnTat 1.1 utilisé pour initier l'infection.

\section{Inoculation des trypanosomes}

Un cryostabilat de AnTat 1.1, un variant antigénique de T. brucei brucei MAVUBWE/66/EATRO 1125 a été réactivé sur souris (2). Au troisième jour, le sang d'une des souris a été prélevé par ponction cardiaque. Ce sang a été dilué dans du tampon PSG jusqu'à obtention d'une suspension de 5 trypanosomes par champ (x 200) et injecté sous la peau derrière l'oreille à raison de $1 \mathrm{ml}$ par bovin.

\section{Prélèvements}

A l'aide d'un tube vacutainer sec, $10 \mathrm{ml}$ de sang ont été prélevés sur chaque animal au niveau de la veine jugulaire avant l'inoculation $\left(\mathrm{J}_{0}\right)$, puis tous les 1-2 jours jusqu'au jour 15 , ensuite 1-2 fois par semaine jusqu'au jour 153 (fin des observations). Le sérum obtenu après centrifugation était réparti en aliquots de $1,5 \mathrm{ml}$ et stocké à $-70^{\circ} \mathrm{C}$. A chaque séance de prélèvement, du sang a également été prélevé dans un tube hépariné pour examen parasitologique.

\section{Analyses}

La parasitémie a été établie en examinant, en contraste de phase entre lame et lamelle, une goutte du buffy coat obtenu après la microcentrifugation (7). La parasitémie a été exprimée en nombre moyen de trypanosomes par champ (x 400) sur vingt champs observés.

La titration des anticorps trypanolytiques AnTat 1.1-spécifiques a été réalisée selon la méthode décrite par Van Meirvenne et coll. (12). Ces tests de trypanolyse ont surtout été effectués pour vérifier si l'inoculation de l'AnTat 1.1 était bien suivie d'infection active.

La détection des antigènes circulants de $T$. brucei a été réalisée par ELISA selon la méthode développée par Nantulya et Lindquist (10) à l'International Livestock Research Institute (ILRI), Nairobi, Kenya. Les réactifs utilisés dans cette expérimentation (anticorps monoclonaux et conjugués) ont été gracieusement fournis par le bureau conjoint FAO/AIEA à Vienne, sous forme d'un kit. Le protocole d'utilisation du kit a été suivi.

\section{RESU LTATS}

\section{Titration des anticorps lytiques AnTat 1.1-spécifiques}

Sauf chez un bovin Borgou, des anticorps trypanolytiques AnTat 1.1-spécifiques ont été mis en évidence chez tous les animaux ino- culés. Avant l'inoculation au $\mathrm{J}_{0}$, aucun des animaux n'a présenté des anticorps dirigés contre AnTat 1.1. Mais ces derniers sont apparus brusquement à partir du jour 7 pour augmenter très rapidement au cours des jours 8 et 9 et se maintenir en plateau jusqu'au jour 44, et descendre lentement par la suite. Aucun sérum des animaux témoins non infectés n'a présenté d'anticorps AnTat 1.1 au cours de l'expérience.

Ces résultats indiquent donc que l'inoculation d'AnTat 1.1 a été suivie d'infection chez tous les bovins Lagunaire et zébus Bororo blancs et chez 9 des 10 bovins Borgou.

\section{Signes cliniques}

Les bovins de races Lagunaire et Borgou infectés ont présenté une maladie sub-clinique ou clinique très bénigne caractérisée par un léger abattement et réduction de l'appétit pendant quelques jours, suivie d'une guérison clinique spontanée en moins de 20-30 jours. Tous les zébus, par contre, ont présenté une maladie à évolution chronique, caractérisée par des hypertrophies ganglionnaires, de la diarrhée intermittente, une dégradation de l'état général avec amaigrissement progressif et apparition de maladies intercurrentes, telle la dermatophilose dans la phase terminale. Aucun des animaux témoins non infectés n'a présenté de signes de trypanosomose.

\section{Parasitémie (buffy coat) et antigénémie (ELISA)}

La cinétique des parasites (nombre moyen de trypanosomes par champ sur 20 champs de buffy coat observés) et des antigènes circulants (DO) est présentée à la figure 1 pour les bovins Lagunaire, dans la figure 2 pour les bovins Borgou et dans la figure 3 pour les zébus Bororo blancs. Les bovins Lagunaire et Borgou ont présenté une évolution très similaire de la parasitémie et de l'antigénémie. Dans ces deux races, la parasitémie devenait positive à partir du jour 5 post-infection, présentait deux pics successifs aux jours 5-7 et jours 9-10, et s'estompait par la suite après le jour 15. Quarantequatre jours après l'infection, le trypanosome n'était plus décelable sur aucun des sujets Lagunaire ou Borgou infectés. Ils sont tous restés négatifs jusqu'à la fin de la période d'observation. Chez les bovins zébus Bororo blancs, la parasitémie devenait positive à partir du jour 4 post-infection, présentait deux pics vagues aux environs des jours 6 et 10 et devenait par la suite intermittente et irrégulière jusqu'à la fin de la période d'observation. Tous les sujets témoins non inoculés des trois races sont restés négatifs pendant toute la période d'observation.

Les figures 1 et 2 indiquent que chez les bovins Lagunaire et Borgou les antigènes circulants étaient détectables avec des valeurs de DO supérieures à 0,050 (seuil de positivité indiqué par l'ILRI) à partir des jours 7 et 8 post-infection seulement, c'est-àdire immédiatement après le premier pic parasitaire. Après le deuxième pic parasitaire, ils sont devenus facilement détectables chez la plupart des sujets jusqu'aux environs du jour 37. Par la suite, les antigènes circulants ont régressé rapidement chez la plupart des sujets et sont devenus indétectables après le jour 44.

La figure 3 montre clairement que chez les zébus Bororo blancs, aux parasitémies nettement plus faibles que chez les bovins Borgou et Lagunaire, l'antigénémie est restée en dessous du seuil de détection pendant pratiquement toute la durée de l'infection ; seuls quelques sujets ont présenté des antigénémies très faiblement positives juste après le premier ou le deuxième pic parasitaire.

Dans le tableau I, les pourcentages de positivité obtenus par détection de parasites, par détection des antigènes circulants et en combinant les deux tests, sont comparés respectivement pour les bovins Borgou, Lagunaire, les zébus Bororo blancs et les trois 


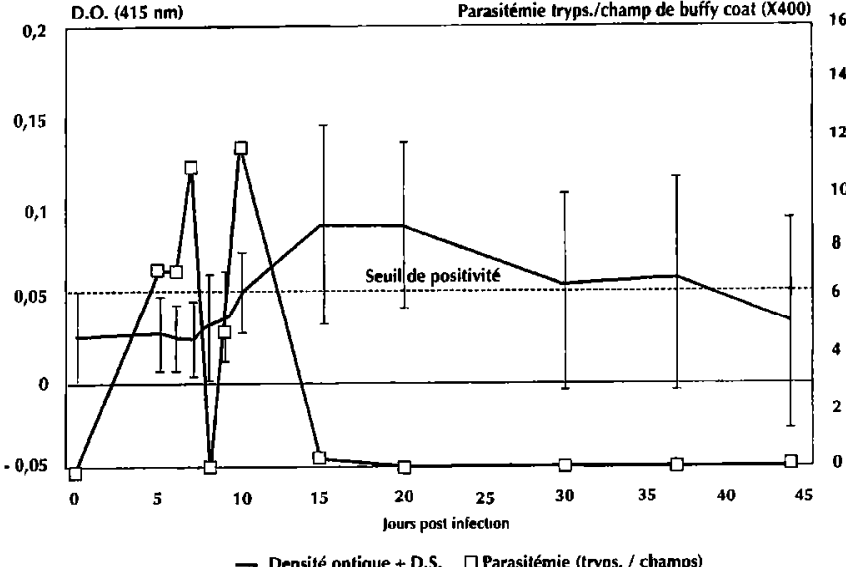

Figure 1 : infection expérimentale à T. b. brucei chez les bovins Lagunaire $(n=10)$ : cinétique des parasites et des antigènes circulants.

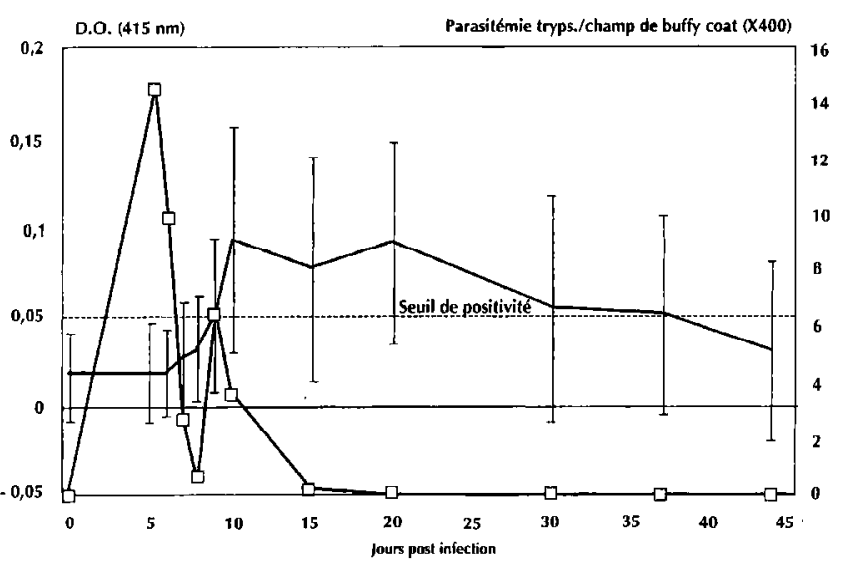

- Densité optique + D.S. 口Parasitémie (tryps. / champs)

Figure 2 : infection expérimentale à T. b. brucei chez les bovins Borgou $(n=9)$ : cinétique des parasites et des antigènes circulants.

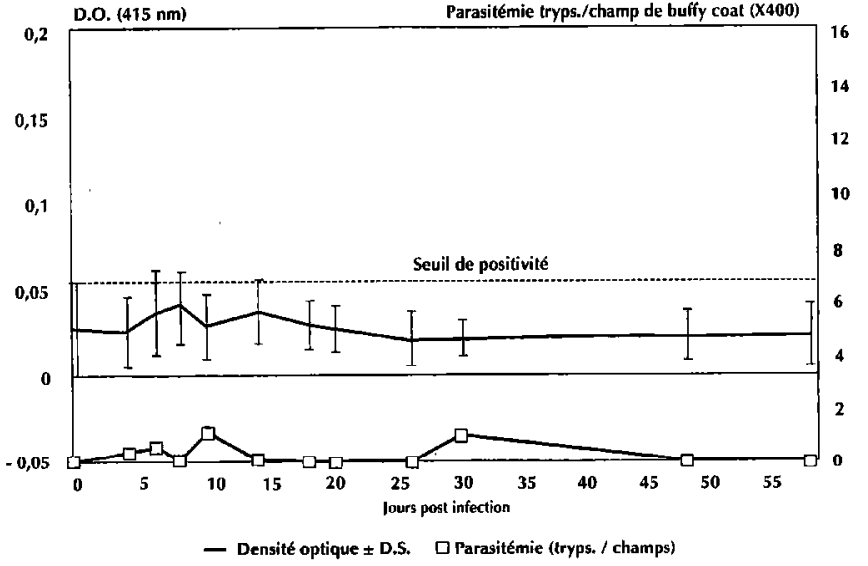

Figure 3 : infection expérimentale à $\mathrm{T}$. b. brucei chez les zébus Bororo blancs $(n=10)$ : cinétique des parasites et des antigènes circulants.

races confondues. Pour les trois races confondues, au cours de la période d'infection active, 40,24 p. 100 des échantillons ont été positifs pour la parasitémie et seulement 20,46 p. 100 pour l'antigénémie. Le pourcentage de positivité obtenu par détection des antigènes circulants était particulièrement bas $(4,09$ p. 100) chez les bovins zébus Bororo blancs aux parasitémies très basses (figure 3). Le tableau I indique encore que les deux tests combinés ont permis de détecter 50,15 p. 100 des infections actives.

\section{DISCUSSION}

Au $\mathrm{J}_{0}$, l'absence d'anticorps trypanolytiques AnTat 1.1-spécifiques chez tous les sujets de l'expérience, infectés et témoins non infectés, semble bien indiquer qu'aucun des animaux n'avait eu un contact récent avec ce variant. Ce variant avait précisément été retenu par Demey (2) pour initier l'infection expérimentale en raison de son absence habituelle dans les infections naturelles chez les ruminants domestiques en Afrique. L'apparition brusque des anti- corps trypanolytiques AnTat 1.1-spécifiques à partir du jour 7 chez 29 des 30 animaux inoculés signale l'infection de ces animaux par AnTat 1.1. Un bovin Borgou n'ayant pas développé d'anticorps AnTat 1.1-spécifiques a été considéré comme n'ayant pas développé d'infection active et n'est, par ailleurs, jamais apparu comme positif, ni pour la parasitémie, ni pour l'antigénémie. Les données de cet animal n'ont donc pas été retenues pour le calcul de la sensibilité des deux méthodes de diagnostic utilisées. Pour tous les autres sujets infectés, les prélèvements retenus pour le calcul de la sensibilité des méthodes de diagnostic sont ceux qui vont du premier jusqu'au dernier jour de positivité de la parasitémie chez chaque individu considéré séparément. Au cours de cette période, il est en effet certain que l'infection trypanosomienne était active.

Le comportement clinique des animaux est très variable suivant les races. Bien que tous les bovins Lagunaire et Borgou aient eu une maladie sub-clinique ou clinique, celle-ci était bénigne et suivie de guérison spontanée. Tous les zébưs Bororo blancs infectés, par contre, ont développé une maladie clinique caractéristique, évoluant de façon chronique avec dégradation de l'état général, amaigrissement progressif et éventuellement apparition de maladies intercurrentes au cours de la phase terminale. Ceci était associé à une parasitémie d'intensité faible mais prolongée (figure 3).

Pour l'ensemble des observations, la sensibilité des méthodes était de 40,24 p. 100 pour le buffy coat, 20,46 p. 100 pour la détection des antigènes de $T$. brucei et 50,15 p. 100 pour les deux tests combinés. Pour la détection de l'infection à T. brucei, la sensibilité de la méthode du buffy coat paraît donc nettement supérieure à celle de la détection des antigènes circulants par ELISA. Toutefois, de très fortes variations raciales ont également été observées. Chez les bovins Borgou et Lagunaire qui ont développé des parasitémies fortes mais très courtes la sensibilité des deux tests était nettement meilleure que chez les zébus qui, eux, ont développé des parasitémies beaucoup plus faibles mais prolongées. Ainsi, chez les zébus Bororo blancs la sensibilité du test de détection des antigènes était réduite à 4,09 p. 100. La figure 3 montre que chez ces derniers la DO observée au cours de l'infection est pratiquement toujours restée en-dessous du seuil de positivité établi par l'ILRI. La relation entre le faible taux de positivité de l'antigénémie et les parasitémies très faibles chez les zébus paraît évidente.

Augmenter la sensibilité du test de détection des antigènes circulants en abaissant le seuil de positivité jusqu'à, par exemple, 0,025 
Tableau I

Infection expérimentale à T. b. brucei de bovins Borgou $(n=9)$, Lagunaire $(n=10)$ et zébus Bororo blancs $(n=10)$ : comparaison des pourcentages de positivité obtenus par détection des parasites, des antigènes circulants et en combinant les deux tests

\begin{tabular}{|c|c|c|c|c|}
\hline & $\begin{array}{l}\text { Bovins } \\
\text { Borgou }\end{array}$ & $\begin{array}{c}\text { Bovins } \\
\text { Lagunaire }\end{array}$ & $\begin{array}{c}\text { Bovins } \\
\text { zébus Bororo } \\
\text { blancs }\end{array}$ & Total \\
\hline Parasitémie positive & $\begin{array}{c}48,14 \% \\
(81)\end{array}$ & $\begin{array}{c}45,55 \% \\
(90)\end{array}$ & $\begin{array}{c}32,80 \% \\
(189)\end{array}$ & $\begin{array}{c}40,24 \% \\
(333)\end{array}$ \\
\hline Antigénémie positive & $\begin{array}{c}44,44 \% \\
(81)\end{array}$ & $\begin{array}{c}30 \% \\
(90)\end{array}$ & $\begin{array}{c}4,09 \% \\
(171)\end{array}$ & $\begin{array}{c}20,46 \% \\
(342)\end{array}$ \\
\hline Tests combinés & $\begin{array}{c}70,37 \% \\
(81)\end{array}$ & $\begin{array}{c}60 \% \\
(90)\end{array}$ & $\begin{array}{c}34,56 \% \\
(162)\end{array}$ & $\begin{array}{c}50,15 \% \\
(333)\end{array}$ \\
\hline
\end{tabular}

( ) : nombre d'échantillons examinés et retenus pour les calculs des positivités.

paraît impossible sans altérer la spécificité du test. En effet, chez les différents animaux témoins non infectés, comme chez tous les infectés au jour 0, avant l'infection (figures 1, 2 et 3), la moyenne des $\mathrm{DO}$ observées était déjà de $0,025 \pm 0,049$. De plus, il y a déjà des faux positifs chez les animaux non infectés, même en adoptant le seuil de positivité de 0,05 recommandé dans le kit.

Il n'a pas été possible de confirmer les résultats de Nantulya $(8,9)$ et de Nantulya et coll. $(10,11)$ qui ont trouvé le test de détection des antigènes circulants plus sensible que la méthode du buffy coat (7). Les résultats de cette étude sur le test de détection des antigènes de $T$. brucei, plus particulièrement chez les bovins zébus Bororo blancs à parasitémies faibles, se rapprochent des observations de Kanwe et coll. (6), de Desquesnes et de la Rocque (3) et de Faye (5) sur le test de détection des antigènes de $T$. vivax pour lequel ils ont observé respectivement une sensibilité de 9,9,2,1 et 11 p. 100 seulement.

Chez les bovins Lagunaire et Borgou (figures 1 et 2), qui ont éliminé spontanément les parasites en moins de 44 jours, on remarque clairement que la méthode de détection des antigènes donne des faux négatifs en début d'infection et des faux positifs en fin d'infection. Tout se passe comme si la détection de l'antigénémie était différée de quelques jours par rapport à celle de la parasitémie. Selon Nantulya et Lindqvist (10) et Desquesnes et de la Rocque (3), les antigènes recherchés étant internes, le test pourrait rester négatif pendant les premières périodes de l'infection (10 à 20 jours), jusqu'à ce qu'une quantité importante d'antigènes soit libérée par lyse des parasites. Après traitement, le test resterait encore positif jusqu'à une quinzaine de jours.

La combinaison des deux tests permet d'augmenter le pourcentage d'infections actives diagnostiquées pour atteindre respectivement 70,37 p. 100 chez les Borgou, 60 p. 100 chez les Lagunaire, 34,56 p. 100 chez les zébus Bororo blancs et 50,15 p. $100 \mathrm{chez}$ les trois races confondues. L'intérêt de combiner les deux tests reste marginal.

Chez les animaux à parasitémie faible, en particulier, le pourcentage d'infections actives diagnostiquées reste en-dessous des limites acceptables.

\section{CONCLUSION}

Le test de détection des antigènes circulants à l'aide d'anticorps monoclonaux anti-T. brucei a été évalué au Bénin dans des condi- tions d'infection expérimentale standardisée chez des bovins réputés trypanotolérants (Lagunaire et Borgou) et trypanosensibles (zébus Bororo blancs). Pour tous les animaux confondus, la sensibilité du test a été de 20,46 p. 100, nettement inférieure à celle du buffy coat (40,24 p. 100). La sensibilité du test varie fortement en fonction du type d'animaux infectés ; elle est la plus élevée chez les bovins Borgou aux parasitémies expérimentales élevées et courtes $(44,44$ p. 100$)$ et la plus faible chez les zébus Bororo blancs aux parasitémies faibles, intermittentes et prolongées (4,09 p. 100).

Les faux négatifs en début d'infection et les faux positifs après infection compromettent également la valeur du test pour le diagnostic des infections actives. Pour diagnostiquer les infections actives à $T$. brucei chez les animaux à très faible parasitémie, il faut recourir au test d'inoculation des animaux de laboratoire ou à la trousse KIVI (kit for in vitro isolation) (1).

\section{Remerciements}

Les auteurs remercient le bureau conjoint FAO/AIEA à Vienne et l'ILRI (ex-ILRAD) pour le don des réactifs d'ELISA de détection des antigènes circulants de T. brucei, l'Union européenne pour le financement des études à travers le projet TS2-0076-B(GDF), ainsi que M. Rudy Baelmans pour son assistance technique.

\section{BIBLIO GRAPHIE}

1. AERTS D., TRU C P., PEN CHENIER L., CLAES Y., LE RAY D., 1992. A kit for in vitro isolation of trypanosomes in the field: first trial with sleeping sickness patients in the Congo Republic. Trans. R. Soc. trop. Med. Hyg., 86: 394-395.

2. DEMEY F., 1987. Contribution à l'étude des paramètres pour l'identification et la sélection d'animaux trypanotolérants. Thèse PhD2, Institut de Médecine tropicale, Anvers, Belgique, 201 p.

3. DESQ UESNES M., de La RO CQUE S., 1995. Comparaison de la sensibilité du test de Woo et d'un test de détection des antigènes de Trypanosoma vivax chez deux moutons expérimentalement infectés avec une souche guyanaise du parasite. Revue Elev. Méd. vét. Pays trop., 48 : 247-253.

4. DOKO A.S., GUEDEGBE B., BAELMANS R., DEMEY F., N'DIAYE A., PANDEY V.S., VERHULST A., 1991. Trypanosomiasis in different breeds of cattle from Benin. Vet. Parasitol., 40: 1-7. 
5. FAYE J., 1993. The use of an antigen-detection ELISA for the diagnosis of trypanosome infection in N'Dama cattle in Gambia. In: Improving the diagnosis and control of trypanosomiasis and other vector-borne diseases of African livestock using immunoassay methods. Vienna, Austria, IAEA, p. 51-57. (IAEA-TECD O C-707)

6. KANWE A.B., BENGALY Z., SAULNIER D., DUVALLET G., 1992. Evaluation du test de détection des antigènes circulants de trypanosomes à l'aide d'anticorps monoclonaux. Infections expérimentales et naturelles. Revue Elev. Méd. vét. Pays trop., 45: 265-271.

7. MURRAY M., MURRAY P.K., M CIBTYRE W.I.M.,1977. An improved parasitological technique for the diagnosis of African trypanosomiasis. Trans. R. Soc. trop. Med. Hyg., 40: 267-272.

8. NANTULYA V.M., 1990. Trypanosomiasis in domestic animals: the problems of diagnosis: Revue sci. tech. O ff. int. Epizoot., 9: 357-367.

\section{Summary}

Doko A., Verhulst A., Pandey V.S., Büscher Ph., Lejon V. Detection of blood antigens in Borgu, Lagoon and White Bororo zebu cattle experimentally infected with $T$. brucei brucei

Borgu ( $n=10)$, Lagoon ( $n=10$ ) and White Bororo zebu $(n=10)$ cattle were experimentally infected in Benin with a clone of Trypanosoma brucei brucei for evaluation of blood antigens using ELISA tests. N one of the three non-inoculated control animals for each breed developed any infection. Borgu and Lagoon cattle developed a benign disease followed by spontaneous recovery, whereas White Bororo zebus developed a typical chronic disease, fatal within several months. O verall ELISA sensitivity to detect $\mathrm{T}$. brucei antigens was $20.46 \%$, clearly lower than that of the buffy coat method to detect parasites $(40.24 \%)$. The sensitivity of ELISA tests differed greatly according to the type of animals and the dynamics of infection. It was highest in Borgu cattle $(44.44$ $\%)$, which had high parasitaemias of short duration and lowest in White Bororo zebus (4.09\%), which had low and intermittent parasitaemias. To increase the sensitivity of ELISA tests by decreasing the cut-off point of optical density (threshold of positivity) from 0.050 to 0.025 would highly compromise the test specificity. The false negatives at the beginning of the infection and false positives after spontaneous cure further compromise the validity of ELISA tests for diagnosis of active $T$. brucei infections.

Key words: Borgu cattle - Lagoon cattle - W hite Fulani zebu Trypanosoma brucei brucei - Experimental infection - ELISA Antigen - Benin.
9. NANTU LYA V.M.,1991. M olecular diagnosis of parasites. Experientia, 47: $142-145$

10. NANTULYA V.M., LINDQ VIST K.J.,1989. Antigen-detection enzyme immunoassays for the diagnosis of Trypanosoma vivax, $T$. congolense and T. brucei infections in cattle. Trop. Med. Parasitol., 40: 267-272.

11. NANTULYA V.M., MUSOKE A.J., RURANGIRWA F.R., SAIGAR N., MINJA S.H., 1987. M onoclonal antibodies that distinguish Trypanosoma congolense, T. vivax and T. brucei. Parasite Immunol., 9: 421-431.

12. VAN MEIRVENNE N., JANSSEN S P.G., MAGNUS E., 1975. Antigenic variation in syringe passaged populations of Trypanosoma (Trypanozoon) brucei. 1. Rationalization of the experimental approach. Ann. Soc. Belge Méd. trop., 55: 1-23.

Reçu le 17.7.96, accepté le 14.10 .96

\section{Resumen}

Doko A., Verhulst A., Pandey V.S., Büscher Ph., Lejon V. Detección de antígenos circulantes durante una infección experimental por Trypanosoma brucei brucei en bovinos Borgou, Lagunare y cebúes Bororo blancos

Se infectaron experimentalmente bovinos Borgou ( $n=10)$, Lagunare $(n=10)$ y cebúes Bororo blancos $(n=10)$ de Benin, con un clon de Trypanosoma brucei brucei, con el fin de evaluar el test ELISA para la detección de antígenos circulantes. Ninguno de los tres testigos no inoculados de cada una de las razas desarrolló la infección. Los bovinos lagunares y Borgou desarrollaron una enfermedad benigna, seguida por una cura espontánea, los cebúes Bororo blancos presentaron una enfermedad tipica con apariencia crónica, fatal al cabo de varios meses. Para todos los animales en general, la sensibilidad del test de detección de antígenos circulantes de $T$. brucei fue de 20,46 p. 100 , francamente inferior a la de la detección de los parásitos mediante el método de buffy coat $(40,24$ p. 100). La sensibilidad del test varía fuertemente en función del tipo de animales infectados y de la apariencia de la infección ; siendo más alta en los bovinos Borgou con parasitemias altas, pero de menor duración $(44,44$ p. 100) y más baja en los cebúes Bororo blancos, con parasitemias bajas e intermitentes $(4,09$ p. 100). La posibilidad de aumentar la sensibilidad del test de detección de antígenos circulantes mediante una disminución de la densidad óptica de 0,050 (límite de positividad) a 0,025 sin alterar la especificidad parece imposible. En comparación con el buffy coat, los falsos negativos al inicio de la infección y los falsos positivos después de la cura espontánea comprometen de forma similar el valor del test para el diagnóstico de las infecciones activas por T. brucei.

Palabras clave : Bovino Borgou - Bovino des Lagunes - Cebú White Fulani - Trypanosoma brucei brucei - infección experimental - ELISA - Antígeno - Benin. 